\title{
URBAN FORESTRY IN MOGI GUAÇU, SÃO PAULO STATE, BRAZIL
}

\author{
Laerte Scanavaca Júnior ${ }^{1}$, Rony Felipe Marcelino Corrêa ${ }^{2}$ \\ ${ }^{1}$ EMBRAPA - Brazilian Agricultural Research Corporation, Brazil. E-mail: laerte.scanavaca@usp.br \\ ${ }^{2}$ UFSCar - Federal University of São Carlos, Brazil. E-mail: ronycorrea@ hotmail.com
}

\section{ABSTRACT}

This study evaluated urban forestry in Mogi Guaçu, São Paulo State, Brazil, according to purchasing power. Stratified sampling was carried out in five neighborhoods, totaling 75 blocks, enabling the identification of 57 species, 20 of which are native and 37 exotics. The frequency of exotic specimens was $77.15 \%$. The three most frequent species $(57.87 \%)$ had the highest phytosanitary occurrences. Small trees presented $63.13 \%$, medium ones $29.33 \%$ and large trees with $7.54 \%$ of frequency. Mogi Guaçu had an average Green Area Index (GAI) of $10.85 \mathrm{~m}^{2}$ per inhabitant, with good potential to increase at low cost, as there are many empty spaces and the replacement of trees that presented problems must be with large trees of native forest near the city. There was no correlation between purchasing power and GAI.

Keywords: Plant health, green area index (GAI), conflicts, species diversity, ecological benefits

\section{FLORESTA URBANA EM MOGI GUAÇU, ESTADO DE SÃO PAULO, BRASIL}

\section{RESUMO}

Este estudo avaliou a arborização urbana em Mogi Guaçu, São Paulo, Brasil, de acordo com o poder aquisitivo. A amostragem estratificada foi realizada em cinco bairros, totalizando 75 blocos, possibilitando a identificação de 57 espécies, sendo 20 nativas e 37 exóticas. A frequência de espécimes exóticos foi de 77,15\%. As três espécies mais frequentes $(57,87 \%)$ apresentaram as maiores ocorrências fitossanitárias. Árvores pequenas apresentaram 63,13\%, as médias 29,33\% e as árvores grandes com 7,54\% da frequência. Mogi Guaçu possuía um índice de área verde (IAV) médio de $10,85 \mathrm{~m}^{2}$ por habitante, com bom potencial para aumentar a baixo custo, pois existem muitos espaços vazios e a substituição das árvores que apresentaram problemas deve ser com árvores de grande porte da floresta nativa nas proximidades da cidade. Não houve correlação entre poder aquisitivo e o IAV.

Palavras-chave: Fitossanidade, índice de área verde (IAV), conflitos, diversidade de espécies, benefícios ecológicos 


\section{INTRODUCTION}

In Brazil, more than $84 \%$ of the population lives in urban centers, in the state of São Paulo, Brazil, the urban population is over 93\% (IBGE, 2019). Cities are heat islands due to the low reflectivity materials used, low permeability, pollution, etc. Urban forestry can alleviate these problems by purifying the air, absorbing heavy metals, reducing the risk of flooding, absorbing noise pollution, etc. (HOLDER \& GIBBES, 2017; LIVESLEY et al., 2016; McPHERSON et al., 2016; RUSSO et al., 2016; SANUSI et al., 2017; SU et al., 2016; WANG et al., 2018; YANG et al., 2019).

Trees require maintenance and pruning when they conflict with urban infrastructure, which generates some costs, however, all studies have shown that the benefits outweigh the costs at the rate of 1.52 to 6.13 per dollar invested (McPHERSON et al., 2016; WANG et al., 2018). The rate of return depends on many factors, the most important of which are the concentration of pollutants and the volume of the treetops.

The World Health Organization (WHO) recommends a minimum vegetation area of $12 \mathrm{~m}^{2}$ per inhabitant for a good quality of life, and few Brazilian municipalities reach this index. In Brazil, there is no master plan for the country, states or municipalities, although the legislation obliges cities with more than 20 thousand inhabitants to have an Urban Forestry Master Plan, however the majority of city halls are not concerned with this. The objective of this work was to conduct a stratified research in five neighborhoods in the municipality of Mogi Guaçu, São Paulo State, Brazil, to study the influence of purchasing power on urban forestry; frequencies of native and exotic species and occurrence of pests and diseases depending on their origin.

\section{MATERIAL AND METHODS}

Mogi Guaçu founded in 1877, is located in the region of Campinas, in the state of São Paulo, Brazil, 22 $22^{\prime} 15^{\prime}$ ' S, 46 56'16' W, average altitude of $587 \mathrm{~m}$ and average annual precipitation of $1,240.50 \mathrm{~mm}$. With a population of 151,888 inhabitants, spread over an area of $812,753 \mathrm{~km}^{2}$, with a demographic density of 168.99 inhabitants per $\mathrm{km}^{2}$ and HDI of 0.774 (IBGE, 2019).

The municipality has five industrial districts with a diversified profile, companies in the paper and cellulose, food, metallurgy and cosmetics sectors, among others. The climate is tropical in altitude with dry winter, minimum average temperature of $15.1^{\circ} \mathrm{C}$ and maximum of $27.9^{\circ} \mathrm{C}$. It 
presents $84.7 \%$ of the population between the middle and upper classes and $15.3 \%$ in the lower class (IBGE, 2019).

Stratified sampling was carried out in five neighborhoods, Jardim Planalto Verde (high standard), with an area of 375,804.9 $\mathrm{m}^{2}$, Jardim Centenário (high to medium standard), with an area of 397,338.8 $\mathrm{m}^{2}$, Jardim Novo I (Medium Standard), with an area of 686,561.1 $\mathrm{m}^{2}$, Jardim Ypê I (low to medium standard) with an area of 233,831.7 $\mathrm{m}^{2}$, and Jardim Santa Terezinha (low standard) with an area of 261,274.5 $\mathrm{m}^{2}$. Fifteen blocks were inventoried per neighborhood. Neighborhood maps were used for sampling, using AutoCad software and spreadsheets for data collection.

The data were collected by a trained person, covering the five neighborhoods, totaling 75 blocks, the four sidewalks on each block.

The identification of the species, according to the classification of Engler, was done through the personal knowledge of the team. For the species not identified in the field, the available vegetative material (leaves, flowers and fruits) was collected, photographed and taken to Embrapa Meio Ambiente in Jaguariúna, São Paulo State, Brazil, for identification with the help of botanists.

In the field research, species were identified from the common name and some qualitative parameters were analyzed, such as plant health, conflicts, height, etc.

\section{RESULTS AND DISCUSSION}

Fifteen blocks per neighborhood were evaluated, totaling 1,299 trees, 49 failures and 27 unidentified trees, 57 species, 20 natives and 37 exotics, 24 families, $22.85 \%$ native and $77.15 \%$ exotic. Three species with more than $10 \%$ of the population: Licania tomentosa (native) with 12.95\%; Ligustrum lucidum (exotic) with $25.18 \%$ and Murraya exotica (exotic) with 19.74\%, which corresponds to $57.87 \%$ of the population (Table 1). Similar results were found by other researchers in the number of species, predominance of exotic species and high frequency of few species (AVOLIO et al., 2018; BLOOD et al., 2016; MORGENROTH et al., 2016; NOWAK et al., 2016; OLIVEIRA et al., 2018; WANG et al., 2018). Worldwide, urban forestry is concentrated in some predominantly exotic species. The main reasons for this practice to occur are the low availability of seeds, the knowledge of these few traditional species, associated with the fear of taking risks with unknown species. 
The two families with the highest frequencies were Oleaceae with $34.64 \%$ and Rutaceae with 20.94\%; with presented the highest percentages of phytosanitary problems. The Rutaceae family presented the largest attack of pests (73.87\%), pests and diseases $(53.23 \%)$, pests and mechanical damage (60\%). The Oleaceae family presented the greatest attacks of diseases (33.33\%), mechanical damage (24\%), diseases and mechanical damage $(80.95 \%)$ and pests, diseases and mechanical damage with $79.49 \%$ of attacks. Although the species are exotic, the high frequency favored the highest percentages of attacks (Table 1).

The increase in species diversity increases the risk of infestation of pests and diseases due to the greater opportunity for attack, and at the same time it decreases by diluting with the largest number of species until reaching equilibrium, with 30 to 35 species of trees. From that number, the greater the diversity, the less the infestation of pests and diseases. This is because the number of trees not attacked by pests increases by the most effective dilution and by the physical and chemical masking done by non-host species (Table 1). The increase in all trophic levels makes the community more resistant to biotic and abiotic disorders (DASSOU \& TIXIER, 2016; FIELD et al., 2020; GUO et al., 2019; GUYOT et al., 2015; JACTEL et al., 2019; KLAPWIJK et al., 2016).

In the evolutionary process, species select their pollinators and dispersers (symbiosis). In this way, native species are more interesting than exotic species, because they will attract the fauna associated with them, making the environment more pleasant. In the case of Mogi Guaçu, Seasonal semideciduous forest, forest formation of the atlantic forest, of the species Astronium spp., Schinus spp., Aspidosperma spp., Handroanthus sp., Jacaranda spp., Cordia spp., Parapiptadenia rigida, Peltophorum dubia, Cedrela spp., Copaifera langsdorffii, etc. present in Parque São Marcelo, belonging to the municipality of Mogi Guaçu, would be more appropriate (Table 2).

The trees were in good condition in more than $90 \%$ of the population (Table 3 ). There were few conflicts with the city infrastructure, the main one being with roots (17.55\%), which in general can be easily corrected (Table 4, 5 and 6); 968 trees were pruned, 957 of which were incorrect (Table 7). The problem is serious because 820 trees are small, and theoretically would not need to be pruned or, at most, an orientation pruning. This problem will be corrected by training the pruning team. 
Table 1. Plant health by family in Mogi Guaçu, São Paulo State, Brazil from 02/02/14 to $11 / 30 / 2014$.

\begin{tabular}{|c|c|c|c|c|c|c|c|c|c|}
\hline \multirow[b]{2}{*}{ Family } & \multicolumn{8}{|c|}{ Plant health } & \multirow[b]{2}{*}{ Total } \\
\hline & 1 & 2 & 3 & 4 & 23 & 24 & 34 & 234 & \\
\hline Not identified & 13 & 2 & 2 & 3 & 0 & 1 & 4 & 2 & 27 \\
\hline Anarcadiaceae & 28 & 1 & 2 & 4 & 0 & 1 & 0 & 2 & 38 \\
\hline Annonaceae & 1 & 0 & 0 & 0 & 0 & 0 & 0 & 0 & 1 \\
\hline Apocynaceae & 10 & 1 & 1 & 0 & 0 & 0 & 0 & 0 & 12 \\
\hline Arecaceae & 26 & 0 & 0 & 0 & 0 & 0 & 0 & 0 & 26 \\
\hline Bignoniaceae & 23 & 6 & 1 & 4 & 0 & 0 & 6 & 1 & 41 \\
\hline Buxaceae & 0 & 0 & 1 & 0 & 0 & 0 & 0 & 0 & 1 \\
\hline Chrysobalanaceae & 146 & 8 & 6 & 10 & 4 & 0 & 2 & 2 & 178 \\
\hline Combretaceae & 5 & 1 & 0 & 2 & 1 & 0 & 1 & 2 & 12 \\
\hline Euphorbiaceae & 2 & 0 & 0 & 0 & 0 & 0 & 0 & 0 & 2 \\
\hline Fabaceae & 17 & 0 & 1 & 2 & 0 & 0 & 1 & 0 & 21 \\
\hline Lythraceae & 55 & 11 & 3 & 5 & 1 & 0 & 5 & 4 & 84 \\
\hline Malpighiaceae & 6 & 0 & 1 & 0 & 0 & 0 & 0 & 0 & 7 \\
\hline Malvaceae & 39 & 1 & 0 & 1 & 3 & 0 & 0 & 1 & 45 \\
\hline Melastomataceae & 2 & 0 & 1 & 0 & 0 & 0 & 0 & 4 & 7 \\
\hline Moraceae & 3 & 9 & 1 & 2 & 6 & 0 & 3 & 10 & 134 \\
\hline Myrtaceae & 15 & 1 & 3 & 2 & 0 & 0 & 0 & 0 & 21 \\
\hline Oleaceae & 87 & 4 & 14 & 12 & 14 & 0 & 102 & 217 & 450 \\
\hline Oxalidaceae & 1 & 0 & 0 & 0 & 0 & 0 & 0 & 0 & 1 \\
\hline Pinaceae & 2 & 0 & 0 & 0 & 0 & 0 & 0 & 0 & 2 \\
\hline Proteaceae & 1 & 0 & 0 & 0 & 0 & 0 & 0 & 0 & 1 \\
\hline Rosaceae & 0 & 1 & 0 & 0 & 0 & 0 & 0 & 0 & 1 \\
\hline Rutaceae & 52 & 147 & 5 & 3 & 33 & 3 & 1 & 28 & 272 \\
\hline Solanaceae & 2 & 6 & 0 & 0 & 0 & 0 & 0 & 0 & 8 \\
\hline Verbenaceae & 6 & 0 & 0 & 0 & 0 & 0 & 1 & 0 & 7 \\
\hline Total & 542 & 199 & 42 & 50 & 62 & 5 & 126 & 273 & 1,299 \\
\hline
\end{tabular}

$1=$ healthy; 2 = pest attack; $3=$ diseases; $4=$ mechanical damage; $23=$ pests and diseases; $24=$ pests and mechanical damage; $34=$ diseases and mechanical damage; $234=$ pests, diseases and mechanical damage. 
Table 2. Phenology of the plant by family in Mogi Guaçu, São Paulo State, Brazil from 02/02/14 to $11 / 30 / 2014$.

\begin{tabular}{|c|c|c|c|c|c|c|}
\hline \multirow[b]{2}{*}{ Family } & \multicolumn{6}{|c|}{ Phenology } \\
\hline & 1 & 2 & 3 & 4 & 5 & 6 \\
\hline Not identified & 27 & 0 & 0 & 0 & 0 & 0 \\
\hline Anarcadiaceae & 35 & 0 & 0 & 0 & 3 & 0 \\
\hline Annonaceae & 0 & 0 & 0 & 0 & 0 & 1 \\
\hline Apocynaceae & 4 & 0 & 0 & 1 & 7 & 0 \\
\hline Arecaceae & 26 & 0 & 0 & 0 & 0 & 0 \\
\hline Bignoniaceae & 26 & 2 & 0 & 1 & 9 & 3 \\
\hline Buxaceae & 1 & 0 & 0 & 0 & 0 & 0 \\
\hline Chrysobalanaceae & 176 & 0 & 0 & 0 & 0 & 2 \\
\hline Combretaceae & 10 & 0 & 0 & 0 & 0 & 2 \\
\hline Euphorbiaceae & 1 & 0 & 0 & 0 & 1 & 0 \\
\hline Fabaceae & 9 & 0 & 0 & 1 & 11 & 0 \\
\hline Lythraceae & 73 & 0 & 0 & 1 & 7 & 3 \\
\hline Malpighiaceae & 5 & 0 & 0 & 0 & 0 & 2 \\
\hline Malvaceae & 16 & 0 & 0 & 0 & 27 & 2 \\
\hline Melastomataceae & 4 & 0 & 0 & 0 & 3 & 0 \\
\hline Moraceae & 19 & 0 & 0 & 0 & 0 & 5 \\
\hline Myrtaceae & 12 & 0 & 0 & 0 & 3 & 6 \\
\hline Oleaceae & 403 & 0 & 0 & 2 & 9 & 36 \\
\hline Oxalidaceae & 0 & 0 & 0 & 0 & 0 & 1 \\
\hline Pinaceae & 2 & 0 & 0 & 0 & 0 & 0 \\
\hline Proteaceae & 0 & 0 & 0 & 0 & 1 & 0 \\
\hline Rosaceae & 1 & 0 & 0 & 0 & 0 & 1 \\
\hline Rutaceae & 83 & 0 & 0 & 16 & 2 & 171 \\
\hline Solanaceae & 0 & 0 & 0 & 0 & 6 & 2 \\
\hline Verbenaceae & 5 & 0 & 0 & 0 & 1 & 1 \\
\hline Total & 948 & 2 & 0 & 22 & 90 & 237 \\
\hline
\end{tabular}

$1=$ leaves only; 2 = flower only; $3=$ only fruit; $4=$ leaf, flower and fruit; $5=$ leaf and flower; $6=$ leaf and fruit. 
Table 3. Condition of the plant by family in Mogi Guaçu, São Paulo State, Brazil from 02/02/14 to $11 / 30 / 2014$.

\begin{tabular}{ccccccc}
\hline Family & $\mathbf{1}$ & $\mathbf{2}$ & $\mathbf{3}$ & $\mathbf{4}$ & $\mathbf{5}$ & Total \\
\hline Not identified & 14 & 10 & 3 & 0 & 0 & 27 \\
Anarcadiaceae & 29 & 7 & 2 & 0 & 0 & 38 \\
Annonaceae & 1 & 0 & 0 & 0 & 0 & 1 \\
Apocynaceae & 10 & 1 & 1 & 0 & 0 & 12 \\
Arecaceae & 26 & 0 & 0 & 0 & 0 & 26 \\
Bignoniaceae & 22 & 17 & 1 & 1 & 0 & 41 \\
Buxaceae & 0 & 1 & 0 & 0 & 0 & 1 \\
Chrysobalanaceae & 154 & 22 & 2 & 0 & 0 & 178 \\
Combretaceae & 6 & 6 & 0 & 0 & 0 & 12 \\
Euphorbiaceae & 2 & 0 & 0 & 0 & 0 & 2 \\
Fabaceae & 14 & 7 & 0 & 0 & 0 & 21 \\
Lythraceae & 56 & 23 & 4 & 1 & 0 & 84 \\
Malpighiaceae & 7 & 0 & 0 & 0 & 0 & 7 \\
Malvaceae & 35 & 10 & 0 & 0 & 0 & 45 \\
Melastomataceae & 1 & 2 & 4 & 0 & 0 & 7 \\
Moraceae & 10 & 20 & 3 & 1 & 0 & 34 \\
Myrtaceae & 14 & 6 & 0 & 1 & 0 & 21 \\
Oleaceae & 98 & 265 & 77 & 9 & 1 & 450 \\
Oxalidaceae & 1 & 0 & 0 & 0 & 0 & 1 \\
Pinaceae & 2 & 0 & 0 & 0 & 0 & 2 \\
Proteaceae & 1 & 0 & 0 & 0 & 0 & 1 \\
Rosaceae & 0 & 1 & 0 & 0 & 0 & 1 \\
Rutaceae & 206 & 51 & 9 & 6 & 0 & 272 \\
Solanaceae & 3 & 5 & 0 & 0 & 0 & 8 \\
Verbenaceae & 5 & 2 & 0 & 0 & 0 & 7 \\
Total & 717 & 456 & 106 & 19 & 1 & 1,299 \\
\hline & 17 & & 0 & 0 \\
\hline
\end{tabular}

$1=$ excellent $2=$ good; $3=$ regular; $4=$ bad; $5=$ terrible. 
Table 4. Conflict with wiring, light post and lighting per family in Mogi Guaçu, São Paulo State, Brazil from 02/02/14 to $11 / 30 / 2014$.

\begin{tabular}{|c|c|c|c|c|c|c|c|c|c|}
\hline \multirow[b]{2}{*}{ Family } & \multicolumn{3}{|c|}{ Wiring } & \multicolumn{3}{|c|}{ Light post } & \multicolumn{3}{|c|}{ Lighting } \\
\hline & 1 & 2 & 3 & 1 & 2 & 3 & 1 & 2 & 3 \\
\hline Not identified & 4 & 3 & 20 & 0 & 0 & 27 & 0 & 0 & 27 \\
\hline Anarcadiaceae & 4 & 4 & 30 & 4 & 0 & 34 & 1 & 0 & 37 \\
\hline Annonaceae & 0 & 0 & 1 & 0 & 0 & 1 & 0 & 0 & 1 \\
\hline Apocynaceae & 0 & 0 & 12 & 0 & 0 & 12 & 0 & 0 & 12 \\
\hline Arecaceae & 0 & 0 & 26 & 0 & 0 & 26 & 0 & 0 & 26 \\
\hline Bignoneacea & 5 & 3 & 33 & 4 & 0 & 36 & 2 & 1 & 38 \\
\hline Buxaceae & 0 & 0 & 1 & 0 & 1 & 1 & 0 & 0 & 1 \\
\hline Chrysobalanaceae & 15 & 20 & 143 & 1 & 0 & 172 & 0 & 0 & 178 \\
\hline Combretaceae & 4 & 4 & 4 & 0 & 5 & 12 & 0 & 0 & 12 \\
\hline Euphorbiaceae & 0 & 0 & 2 & 1 & 0 & 1 & 0 & 0 & 2 \\
\hline Fabaceae & 2 & 1 & 18 & 0 & 0 & 21 & 0 & 0 & 21 \\
\hline Lythraceae & 7 & 2 & 75 & 3 & 0 & 81 & 0 & 0 & 84 \\
\hline Malpighiaceae & 2 & 0 & 5 & 0 & 0 & 7 & 0 & 0 & 7 \\
\hline Malvaceae & 1 & 0 & 44 & 2 & 0 & 43 & 0 & 0 & 45 \\
\hline Melastomataceae & 1 & 2 & 4 & 1 & 0 & 6 & 0 & 0 & 7 \\
\hline Moraceae & 7 & 6 & 21 & 4 & 0 & 30 & 2 & 0 & 32 \\
\hline Myrtaceae & 2 & 1 & 18 & 1 & 1 & 19 & 1 & 0 & 20 \\
\hline Oleaceae & 76 & 77 & 297 & 17 & 12 & 421 & 1 & 2 & 447 \\
\hline Oxalidaceae & 1 & 0 & 0 & 0 & 0 & 1 & 0 & 0 & 1 \\
\hline Pinaceae & 0 & 0 & 2 & 0 & 0 & 2 & 0 & 0 & 2 \\
\hline Proteaceae & 0 & 0 & 1 & 0 & 0 & 1 & 0 & 0 & 1 \\
\hline Rosaceae & 0 & 0 & 1 & 0 & 0 & 1 & 0 & 0 & 1 \\
\hline Rutaceae & 5 & 13 & 254 & 5 & 8 & 259 & 0 & 0 & 272 \\
\hline Solanaceae & 0 & 0 & 8 & 1 & 0 & 7 & 0 & 0 & 8 \\
\hline Verbenaceae & 0 & 0 & 7 & 0 & 0 & 7 & 0 & 0 & 7 \\
\hline Total & 136 & 136 & 1,027 & 47 & 24 & 1,228 & 7 & 3 & 1,289 \\
\hline
\end{tabular}

$1=$ current; 2 = potential (when the tree reaches full development), $3=$ without conflict. 
Table 5. Conflict with traffic signs, construction and vehicles per family in Mogi Guaçu, São Paulo State, Brazil from 02/02/14 to 11/30/2014.

\begin{tabular}{|c|c|c|c|c|c|c|c|c|c|}
\hline \multirow[b]{2}{*}{ Family } & \multicolumn{3}{|c|}{ Traffic signs } & \multicolumn{3}{|c|}{ Construction } & \multicolumn{3}{|c|}{ Vehicles } \\
\hline & 1 & 2 & 3 & 1 & 2 & 3 & 1 & 2 & 3 \\
\hline Not identified & 0 & 0 & 27 & 1 & 2 & 24 & 0 & 0 & 27 \\
\hline Anarcadiaceae & 0 & 0 & 38 & 6 & 0 & 32 & 1 & 0 & 37 \\
\hline Annonaceae & 0 & 0 & 1 & 1 & 0 & 0 & 1 & 0 & 0 \\
\hline Apocynaceae & 0 & 0 & 12 & 0 & 0 & 12 & 0 & 0 & 12 \\
\hline Arecaceae & 0 & 0 & 26 & 0 & 0 & 26 & 0 & 0 & 26 \\
\hline Bignoniaceae & 0 & 0 & 41 & 5 & 0 & 36 & 2 & 0 & 39 \\
\hline Buxaceae & 0 & 0 & 1 & 0 & 0 & 1 & 0 & 0 & 1 \\
\hline Chrysobalanaceae & 0 & 0 & 178 & 12 & 12 & 154 & 4 & 0 & 174 \\
\hline Combretaceae & 0 & 0 & 12 & 2 & 1 & 9 & 0 & 0 & 12 \\
\hline Euphorbiaceae & 0 & 0 & 2 & 0 & 0 & 2 & 0 & 0 & 2 \\
\hline Fabaceae & 0 & 0 & 21 & 1 & 0 & 20 & 0 & 0 & 21 \\
\hline Lythraceae & 1 & 0 & 83 & 0 & 4 & 80 & 1 & 0 & 83 \\
\hline Malpighiaceae & 0 & 0 & 7 & 0 & 0 & 7 & 1 & 0 & 6 \\
\hline Malvaceae & 0 & 0 & 45 & 0 & 0 & 45 & 2 & 0 & 43 \\
\hline Melastomataceae & 0 & 0 & 7 & 0 & 1 & 6 & 0 & 0 & 7 \\
\hline Moraceae & 1 & 0 & 33 & 5 & 4 & 25 & 0 & 1 & 33 \\
\hline Myrtaceae & 0 & 0 & 21 & 1 & 0 & 20 & 1 & 0 & 20 \\
\hline Oleaceae & 2 & 0 & 248 & 24 & 18 & 408 & 12 & 0 & 438 \\
\hline Oxalidaceae & 0 & 0 & 1 & 0 & 0 & 1 & 0 & 0 & 1 \\
\hline Pinaceae & 0 & 0 & 2 & 0 & 0 & 2 & 0 & 0 & 2 \\
\hline Proteaceae & 0 & 0 & 1 & 0 & 0 & 1 & 0 & 0 & 1 \\
\hline Rosaceae & 0 & 0 & 1 & 0 & 0 & 1 & 0 & 0 & 1 \\
\hline Rutaceae & 0 & 0 & 272 & 22 & 6 & 244 & 10 & 0 & 262 \\
\hline Solanaceae & 0 & 0 & 8 & 0 & 0 & 8 & 0 & 0 & 8 \\
\hline Verbenaceae & 0 & 0 & 7 & 0 & 0 & 7 & 0 & 0 & 7 \\
\hline Total & 4 & 0 & 1,295 & 80 & 48 & 1,171 & 35 & 1 & 1,263 \\
\hline
\end{tabular}

$1=$ current; $2=$ potential (when the tree reaches full development), $3=$ without conflict. 
Table 6. Conflict with root, culvert, garage and pedestrian per family in Mogi Guaçu, São Paulo State, Brazil from 02/02/14 to 11/30/2014.

\begin{tabular}{|c|c|c|c|c|c|c|c|c|c|c|c|c|}
\hline \multirow[b]{2}{*}{ Family } & \multicolumn{3}{|c|}{ Root } & \multicolumn{3}{|c|}{ Culvert } & \multicolumn{3}{|c|}{ Garage } & \multicolumn{3}{|c|}{ Pedestrian } \\
\hline & 1 & 2 & 3 & 1 & 2 & 3 & 1 & 2 & 3 & 1 & 2 & 3 \\
\hline Not identified & 4 & 0 & 23 & 0 & 0 & 27 & 4 & 0 & 23 & 5 & 0 & 22 \\
\hline Anarcadiaceae & 6 & 0 & 32 & 0 & 1 & 37 & 8 & 1 & 29 & 9 & 0 & 29 \\
\hline Annonaceae & 0 & 0 & 1 & 0 & 0 & 1 & 0 & 0 & 1 & 0 & 0 & 1 \\
\hline Apocynaceae & 0 & 0 & 12 & 0 & 0 & 12 & 1 & 0 & 11 & 1 & 0 & 11 \\
\hline Arecaceae & 0 & 0 & 26 & 0 & 0 & 26 & 2 & 0 & 24 & 0 & 0 & 26 \\
\hline Bignoneacea & 7 & 0 & 34 & 0 & 0 & 41 & 2 & 1 & 38 & 2 & 0 & 39 \\
\hline Buxaceae & 0 & 0 & 1 & 0 & 0 & 1 & 0 & 0 & 1 & 0 & 0 & 1 \\
\hline Chrysobalanaceae & 21 & 1 & 156 & 0 & 0 & 178 & 20 & 8 & 150 & 5 & 0 & 173 \\
\hline Combretaceae & 4 & 0 & 8 & 0 & 0 & 12 & 1 & 0 & 11 & 1 & 0 & 11 \\
\hline Euphorbiaceae & 0 & 0 & 2 & 0 & 0 & 2 & 1 & 0 & 1 & 0 & 0 & 2 \\
\hline Fabaceae & 3 & 0 & 18 & 0 & 0 & 21 & 1 & 0 & 20 & 2 & 0 & 19 \\
\hline Lythraceae & 9 & 0 & 75 & 0 & 0 & 84 & 5 & 2 & 77 & 4 & 0 & 80 \\
\hline Malpighiaceae & 0 & 0 & 7 & 0 & 0 & 7 & 1 & 1 & 5 & 1 & 0 & 6 \\
\hline Malvaceae & 1 & 0 & 44 & 0 & 0 & 45 & 5 & 3 & 37 & 3 & 0 & 42 \\
\hline Melastomataceae & 3 & 0 & 4 & 0 & 0 & 7 & 2 & 0 & 5 & 0 & 0 & 7 \\
\hline Moraceae & 10 & 0 & 24 & 0 & 0 & 34 & 9 & 2 & 23 & 0 & 0 & 34 \\
\hline Myrtaceae & 1 & 0 & 20 & 0 & 0 & 21 & 3 & 1 & 17 & 2 & 0 & 19 \\
\hline Oleaceae & 144 & 0 & 306 & 1 & 1 & 448 & 99 & 6 & 345 & 21 & 0 & 429 \\
\hline Oxalidaceae & 0 & 0 & 1 & 0 & 0 & 1 & 0 & 0 & 1 & 0 & 0 & 1 \\
\hline Pinaceae & 0 & 0 & 2 & 0 & 0 & 2 & 0 & 0 & 2 & 0 & 0 & 2 \\
\hline Proteaceae & 0 & 0 & 1 & 0 & 0 & 1 & 0 & 0 & 1 & 0 & 0 & 1 \\
\hline Rosaceae & 0 & 0 & 1 & 0 & 0 & 1 & 0 & 0 & 1 & 0 & 0 & 1 \\
\hline Rutaceae & 15 & 0 & 257 & 0 & 0 & 272 & 47 & 4 & 221 & 43 & 1 & 228 \\
\hline Solanaceae & 0 & 0 & 8 & 0 & 0 & 8 & 0 & 0 & 8 & 0 & 0 & 8 \\
\hline Verbenaceae & 0 & 0 & 7 & 0 & 0 & 7 & 1 & 0 & 6 & 0 & 0 & 7 \\
\hline Total & 228 & 1 & 1,070 & 1 & 2 & 1,296 & 212 & 29 & 1,058 & 99 & 1 & 1,199 \\
\hline
\end{tabular}

$1=$ current; 2 = potential (when the tree reaches full development), $3=$ without conflict. 
Table 7. Pruning, size and origin of the plant by family in Mogi Guaçu, São Paulo State, Brazil from $02 / 02 / 14$ to $11 / 30 / 2014$.

\begin{tabular}{|c|c|c|c|c|c|c|c|c|}
\hline \multirow[b]{2}{*}{ Family } & \multicolumn{3}{|c|}{ Pruning } & \multicolumn{3}{|c|}{ Size } & \multicolumn{2}{|c|}{ Origin } \\
\hline & $\mathbf{1}$ & 2 & 3 & 1 & 2 & 3 & 1 & 2 \\
\hline Not identified & 0 & 19 & 8 & 15 & 8 & 4 & 0 & 0 \\
\hline Anarcadiaceae & 1 & 22 & 15 & 12 & 21 & 5 & 32 & 6 \\
\hline Annonaceae & 0 & 0 & 1 & 0 & 0 & 1 & 0 & 1 \\
\hline Apocynaceae & 0 & 2 & 10 & 11 & 1 & 0 & 1 & 11 \\
\hline Arecaceae & 0 & 0 & 26 & 20 & 5 & 1 & 0 & 10 \\
\hline Bignoniaceae & 0 & 21 & 20 & 24 & 9 & 8 & 24 & 17 \\
\hline Buxaceae & 0 & 1 & 0 & 1 & 0 & 0 & 0 & 1 \\
\hline Chrysobalanaceae & 3 & 115 & 60 & 119 & 51 & 8 & 178 & 0 \\
\hline Combretaceae & 0 & 10 & 2 & 2 & 3 & 7 & 12 & 0 \\
\hline Euphorbiaceae & 0 & 0 & 2 & 1 & 1 & 0 & 0 & 2 \\
\hline Fabaceae & 2 & 6 & 13 & 10 & 5 & 6 & 6 & 15 \\
\hline Lythraceae & 2 & 52 & 30 & 66 & 16 & 2 & 0 & 84 \\
\hline Malpighiaceae & 0 & 3 & 4 & 6 & 1 & 0 & 1 & 6 \\
\hline Malvaceae & 0 & 18 & 26 & 42 & 3 & 0 & 2 & 43 \\
\hline Melastomataceae & 0 & 6 & 1 & 4 & 2 & 1 & 7 & 0 \\
\hline Moraceae & 0 & 29 & 5 & 18 & 10 & 6 & 0 & 34 \\
\hline Myrtaceae & 0 & 7 & 14 & 18 & 3 & 0 & 16 & 5 \\
\hline Oleaceae & 6 & 422 & 22 & 196 & 207 & 47 & 0 & 450 \\
\hline Oxalidaceae & 0 & 0 & 1 & 0 & 0 & 1 & 0 & 1 \\
\hline Pinaceae & 0 & 0 & 2 & 1 & 0 & 1 & 0 & 2 \\
\hline Proteaceae & 0 & 0 & 1 & 1 & 0 & 0 & 0 & 1 \\
\hline Rosaceae & 0 & 1 & 0 & 0 & 1 & 0 & 0 & 1 \\
\hline Rutaceae & 2 & 215 & 55 & 238 & 34 & 0 & 0 & 272 \\
\hline Solanaceae & 0 & 1 & 7 & 8 & 0 & 0 & 8 & 0 \\
\hline Verbenaceae & 0 & 7 & 0 & 7 & 0 & 0 & 0 & 7 \\
\hline Total & 16 & 957 & 325 & 820 & 381 & 98 & 287 & 969 \\
\hline
\end{tabular}

Pruning: $1=$ correct pruning; $2=$ incorrect; $3=$ not pruned. Size: $1=$ small (tree height up to $6 \mathrm{~m}$ and canopy diameter up to $3 \mathrm{~m}$ ); $2=$ medium (tree height from 6.1 to $9 \mathrm{~m}$ and canopy diameter up to $7 \mathrm{~m}$ ); $3=$ large (tree over $10 \mathrm{~m}$ tall and canopy over $10 \mathrm{~m}$ in diameter); Origin: $1=$ native; 2 = exotic. 
More than $63 \%$ of the trees are small (Table 7). The benefits of urban trees are in the crowns, where together with the photosynthesis process, toxic gases, heavy metals and dust are absorbed, and oxygen and water are eliminated. Noise absorption is also proportional to the canopy volume, therefore, the larger the tree and, consequently, the canopy, the greater the benefits for the population (COUTTS et al., 2015; LIVESLEY et al., 2016; MARTINI et al., 2017; McPHERSON et al., 2016; MONTEIRO et al., 2019; RUSSO et al., 2016; SANUSI et al., 2017; WANG et al., 2018). Thus, on the sidewalks without wiring, large trees should be planted. A small tree generates US $\$ 44.00$ per year of return for each dollar invested, while a large tree generates US $\$ 140.00$ per year (WANG et al., 2018).

The green area index per inhabitant $\left[(\mathrm{GAI})\left(\mathrm{m}^{2}\right.\right.$ inhabitant $\left.\left.{ }^{-1}\right)\right]$ was calculated based on the area of the canopy of the trees according to their height, $3 \mathrm{~m}^{2}$ of canopy for low trees, $7 \mathrm{~m}^{2}$ for medium height trees and $10 \mathrm{~m}^{2}$ for tall trees were stipulated. The weighted harmonic mean was used to determine the green areas of the neighborhoods, and the population demographic density was considered homogeneous, that is, 168.99 inhabitants per $\mathrm{km}^{2}$ (Table 8).

Three neighborhoods had a GAI above that recommended by the World Health Organization (12 $\mathrm{m}^{2}$ per inhabitants), and the general average was slightly below that recommended $\left(10.85 \mathrm{~m}^{2}\right)$ and is not related to purchasing power population, unlike most cities where, the greater the purchasing power, the higher the GAI (BERTINI et al., 2016; LI et al., 2015; MARTINI et al., 2017; MORGENROTH et al., 2016; ORDÓÑEZ et al., 2019; SANUSI et al., 2017; SONG et al., 2016; STEENBERG et al., 2019; XIAO \&McPHERSON, 2016; YANG et al., 2019).

Typically, the wealthiest neighborhoods have larger green areas and less population density, which allows the planting of trees within the property (MONTEIRO et al., 2019), while in the poorer neighborhoods, in addition to the higher population density, the areas are more waterproof. Possible explanations for why this did not occur in Mogi Guaçu are the low distinction between social classes, $84.7 \%$ of the population are middle to upper class and $15.3 \%$ from low class, and the homogenization of demographic density. 
Table 8. Green area index [(GAI) $\left(\mathrm{m}^{2}\right.$ inhabitant-1)] in the different neighborhoods of Mogi Guaçu, São Paulo State, Brazil.

\section{Neighborhood}

\begin{tabular}{|c|c|c|c|c|c|}
\hline Parameters & P.V. & S.T. & J.N.I & J.Y.I & J.C. \\
\hline In total blocks & 33 & 24 & 31 & 29 & 30 \\
\hline Number of courts assessed & 15 & 15 & 15 & 15 & 15 \\
\hline Neighborhood area $\left(\mathrm{m}^{2}\right)$ & 744,700 & 811,500 & 981,600 & 422,400 & 872,400 \\
\hline Area evaluated $\left(\mathrm{m}^{2}\right)$ & $375,804.9$ & $262,274.5$ & $686,561.1$ & $233,831.7$ & $397,338.8$ \\
\hline Green coverage assessed area (\%) & 50.46 & 32.32 & 69.94 & 55.36 & 45.55 \\
\hline Green neighborhood area $\left(\mathrm{m}^{2}\right)$ & $1,797.46$ & $2,701.11$ & $2,210.47$ & $3,217.12$ & $2,392.97$ \\
\hline Inhabitant in the neighborhood $*$ & 222.92 & 208.24 & 172.16 & 400.07 & 193.71 \\
\hline GAI (m² inhabitant-1) & 8.06 & 12.97 & 12.84 & 8.04 & 12.35 \\
\hline
\end{tabular}

\section{CONCLUSION}

All neighborhoods evaluated in Mogi Guaçu showed low species diversity and high percentage of exotic species.

The larger the canopy area, the greater the environmental benefits; therefore, whenever possible, large trees should be planted. Species diversification is also related to the quality of urban forestry and, the greater the diversity, the lower the risk of attack by pests and diseases.

We recommend diversifying with large species from the Seasonal Semidicidual Forest and training the tree pruning team in Mogi Guaçu. 


\section{REFERENCES}

AVOLIO, M.; PATAKI, D. E.; TRAMMELL, T. L. E.; ENDTER-WADA, J., 2018. Biodiverse cities: the nursery industry, homeowners, and neighborhood differences drive urban tree composition. Ecological Monographs, v.0, p.1-18. Available at: <https://digitalcommons.usu.edu/cgi/viewcontent.cgi?article=2598\&context=envs_facpub> Accessed on: May. 13, 2020.

BERTINI, M. A.; RUFINO, R. R.; FUSHITA, A. T.; LIMA, M. I. S., 2016. Public green areas and urban environmental quality of the city of São Carlos, São Paulo, Brazil. Brazilian Journal of Biology, v.76, p.700-707. Available at: <https://www.scielo.br/pdf/bjb/v76n3/1519-6984bjb-1519-698401515.pdf> Accessed on: May. 10, 2020.

BLOOD, A.; STARR, G.; ESCOBEDO, F.; CHAPPELKA, A.; STAUDHAMMER, C., 2016. How do urban forests compare? Tree diversity in Urban and Periurban Forests of the Southeastern US. Forest, v.7, n.6, 120: doi:10.3390/f7060120. Available at: <https://www.mdpi.com/19994907/7/6/120> Accessed on: Apr. 10, 2020.

COUTTS, A. M.; WHITE, E. C.; TAPPER, N. J.; BERINGER, J.; LIVESLEY, S. J., 2015. Temperature and human thermal comfort effects of street trees across three contrasting street canyon environments. Theory Applied of Climatology, v.124, p.55-68. Available at: <https://link.springer.com/article/10.1007/s00704-015-1409-y> Accessed on: Apr. 12, 2020.

DASSOU, A. G.; TIXIER, P., 2016. Response of pest control by generalist predators to local-scale plant diversity: a meta-analysis. Ecology and Evolution. John Wiley and Sons Ltd. Available at: <https://agritrop.cirad.fr/579782/7/ece31917.pdf> Accessed on: Mar. 12, 2020.

FIELD, E.; GIBBS, M.; JACTEL, H.; BARSOUM, N., 2020. Associational resistance to both insect and pathogen damage in mixed forests is modulated by tree neighbour identity and drought. Journal of Ecology, v.108, p.1511-1522. Available at: <https://besjournals.onlinelibrary.wiley.com/doi/pdfdirect/10.1111/1365-2745.13397> Accessed on: Mar. 16, 2020.

GUO, Q.; FEI, S.; POTTER, K.M.; LIEBHOLD, A. M.; WEN, J., 2019. Tree diversity regulates forest pest invasion. PNAS, v.116, p.7382-7386. Available at: <https://www.pnas.org/content/pnas/116/15/7382.full.pdf> Accessed on: Mar. 16, 2020.

GUYOT, V.; CASTAGNEYROL, B.; VIALATTE, A.; DECONCHAT, M.; SELVI, F.; BUSSOTTI, F.; JACTEL, H., 2015. Tree diversity limits the impact of an invasive forest pest. PLOS ONE. Doi: 10.1371/journal.pone.0136469. Sept 11, 2015. Available at: <https://journals.plos.org/plosone/article/file?id=10.1371/journal.pone.0136469\&type=printa ble> Accessed on: Feb. 17, 2020.

HOLDER, C. D.; GIBBES, C., 2017. Influence of leaf and canopy characteristics on rainfall interception and urban hydrology. Hydrology Science Journal, v.62, p.182-190. Available at:

<https://www.tandfonline.com/doi/pdf/10.1080/02626667.2016.1217414https://www.tandfo nline.com/doi/pdf/10.1080/02626667.2016.1217414> Accessed on: Abr. 2, 2020.

IBGE. Cities. Statistical information, 2019. Brazilian Institute of Geography and Statistics (IBGE). Accessed on: 24/02/2019. Available at: <https://cidades.ibge.gov.br/brasil/sp/mogiguacu/panorama > Accessed on: Apr. 20, 2020 (in Portuguese).

JACTEL, H.; POEYDEBAT, C.; HALDER, I.V.; CASTAGNEYROL, B., 2019. Interactive effects of tree mixing and drought on a primary forest pest. Frontiers in Forest and Global Change, v.2: Article 77. Doi: 10.3389/ffgc.2019.00077. Available at: 
<https://www.frontiersin.org/articles/10.3389/ffgc.2019.00077/full> Accessed on: Mar. 1, 2020.

KLAPWIJK, M. $\quad$ J.; BYLUND, H.; SCHROEDER, M.; BJORKMAN, C., 2016. Forest management and natural biocontrol of insect pests. Forestry, v.89, p.253-262. Available at: <https://pub.epsilon.slu.se/14064/7/klapwijk_m_j_et_al_170221.pdf> Accessed on: Jan. 5, 2020.

LI, X.; ZHANG, C.; LI, W.; KZOVKINA, Y. A.; WEINER, D., 2015. Who lives in greener neighborhoods? The distribution of street greenery and its association with residents' socioeconomic conditions in Hartford, Connecticut, USA. Urban Forestry \& Urban Greening, $\quad$ v.14, p.751-759. Available at: <https://hartfordclimate.files.wordpress.com/2016/12/who-lives-in-greenerneighborhoods.pdf> Accessed on: Abr. 23, 2020.

LIVESLEY, S. J.; McPHERSON, E. G.; CALFAPEITRA, C., 2016. The urban forest and ecosystem services: Impacts on urban water, heat, and pollution cycles at the tree, street, and city scale. Journal of Environmental Quality, v.45, p.119-124. Available at: <https://www.fs.fed.us/psw/publications/mcpherson/psw_2016_mcpherson001_livesley.pdf> Accessed on: Mar. 22, 2020.

MARTINI, A.; BIONDI, D.; BATISTA, A. C., 2017. Urban forest components influencing microclimate and cooling potential. Revista Árvore, v.41, p.1-12. Available at: 〈https://www.scielo.br/pdf/rarv/v41n6/0100-6762-rarv-41-06-e410603.pdf> Accessed on: Mar. 26, 2020.

McPHERSON, E. G.; Van DOORN, N.; GOED, J., 2016. Structure, function and value of street trees in California, USA. Urban Forestry \& Urban Green, v.17, p.104-115. Available at: <https://www.fs.fed.us/psw/publications/mcpherson/psw_2016_mcpherson004.pdf> Accessed on: Mar. 11, 2020.

MONTEIRO, M. V.; HANDLEY, P.; DOICK, K. J., 2019. An insight to the current state and sustainability of urban forests across Great Britain based on i-Tree Eco surveys. Forestry, v.93, p.107-123. Doi:10.1093/forestry/cpz054

MORGENROTH, J.; OSTBERG, C.; BOSCH, C. K.; NIELSEN, A. B.; HAUER, R.; SJOMAN, H.; CHEN, W.; JANSSON, M., 2016. Urban tree diversity - Taking stock and looking ahead. Urban Forestry \& Urban Greening, v.15, p.1-5. Available at: $<$ https://www.researchgate.net/publication/283491634_Urban_Tree_Diversity__Taking_Stock_and_Looking_Ahead> Accessed on: Apr. 16, 2020.

NOWAK, D. J.; HOEHN, R. E.; BODINE, A. R.; GREENFIELD, E. J.; O’NEIL-DUNNE, J., 2016. Urban forest structure, ecosystem services and change in Syracuse, NY. Urban Ecosystems, v.19, p.1455-1477. Available at: <https://link.springer.com/content/pdf/10.1007/s11252-013-0326-z.pdf> Accessed on: Mar. 20, 2020.

OLIVEIRA, A. F.; NEVES, C. L. P.; PEREIRA, G. A.; GARCIA, F. H. S.; COELHO, S. J.; PEREIRA, J. A. A., 2018. Floristic of road Forestry conflicting with the electrical networks: a case study in the southern region of Minas Gerais State. Ornamental Horticulture, v.34, p.277-284. Available at: <https://www.scielo.br/pdf/oh/v24n3/2447-536X-oh-24-030277.pdf> Accessed on: Mar 11, 2020.

ORDÓÑEZ, C.; THRELFALL, C. G.; KENDAL, D.; HOCHULI, D. F.; DAVERN, M.; FULLER, R. A.; REE, R.; LIVESLEY, S. J., 2019. Urban forest governance and decision-making: A systematic review and synthesis of the perspectives of municipal mangers. Landscape and 
Urban Planning, v.189, p.166-180. Available at: <https://www.sciencedirect.com/science/article/abs/pii/S0169204618310673?via\%3Dihub> Accessed on: Mar. 16, 2020.

RUSSO, A.; ESCOBEDO, F. J.; ZERBE, S., 2016. Quantifying the local-scale ecosystem services provided by urban treed streetscapes in Bolzano, Italy. Environmental Science, v.3, p.58-76. Available at: <https://www.researchgate.net/publication/292943550_Quantifying_the_localscale_ecosystem_services_provided_by_urban_treed_streetscapes_in_Bolzano_Italy > Accessed on: Mar. 17, 2020.

SANUSI, R.; JOHNSTONE, D. M.; MAY, P. B.; LIVESLEY, S. J., 2017. Microclimate benefits that different street tree species provide to sidewalk pedestrians relate to differences in Plant Area Index. Landscape and Urban Planning, v.157, p.502-511. Available at: <https://www.sciencedirect.com/science/article/abs/pii/S0169204616301633?via\%3Dihub> Accessed on: Mar. 13, 2020.

SONG, X.; CHANG, K. T.; YANG, L.; SCHEFFRAN, J., 2016. Change in environmental benefits of urban land use and its drivers in Chinese cities, 2000-2010. International Journal of Environmental Research and Public Health, v.13, 535; doi:103.390/ijerph13060535. Available at: <https://www.ncbi.nlm.nih.gov/pmc/articles/PMC4923992/> Accessed on: May. 2, 2020.

STEENBERG, J. W. N.; ROBINSON, P. J.; DUINKER, P. N., 2019. A spatio-temporal analysis of the relationship between housing renovation socioeconomic status, and urban forest ecosystems. Urban Analytics and City Science, v.46, p.1115-1131. Available at: <https://journals.sagepub.com/doi/full/10.1177/2399808317752927>. Accessed on: Mar. 16, 2020.

SU, L.; ZHAO, C.; XU, W.; XIE, Z., 2016. Modelling interception loss using the revised Gash model: a case study in a mixed evergreen and deciduous broadleaved forest in China. Ecohydrology, $\quad$ v.9, p.1580-1589. Available at: <https://www.researchgate.net/publication/301920552_Modelling_interception_loss_using_t he_revised_Gash_model_a_case_study_in_a_mixed_evergreen_and_deciduous_broadleaved _forest_in_China/link/5cb17364a6fdcc1d4991154a/download> Accessed on: Abr. 12, 2020.

XIAO, Q.; McPHERSON, E. G., 2016. Surface water storage capacity of twenty tree species in Davis, California. Journal of Environment Quality, v.45, p.188-198. Available at: <https://www.fs.fed.us/psw/publications/mcpherson/psw_2016_mcpherson002_xiao.pdf> Accessed on: Abr. 16, 2020.

WANG, X.; YAO, J.; YU, S.; MIAO, C.; CHEN, W.; HE, X., 2018. Street trees in a Chinese forest city: Structure, benefits and costs. Sustainability, v.10, n.3, 674 Available at: Doi:10.3390/su10030674. Access? Available at: <https://www.mdpi.com/20711050/10/3/674>. Accessed on: Mar. 16, 2020.

YANG, B.; LEE, D. K.; HEO, H. K.; BIGING, G., 2019. The effects of tree characteristics on rainfall interception in urban areas. Landscape and Ecological Engineering, v.15, p.289-296. Available at: 〈https://link.springer.com/article/10.1007/s11355-019-00383-w> Accessed on Mar. 13, 2020. 